\title{
Long-term survival and quality of life of patients with prolonged postoperative intensive care unit stay: Unmasking an apparent success
}

Mario Gaudino, MD, ${ }^{a}$ Fabiana Girola, MD, ${ }^{a}$ Mariantonietta Piscitelli, MD, ${ }^{a}$ Lorenzo Martinelli, MD, ${ }^{\mathrm{b}}$ Amedeo Anselmi, MD, ${ }^{a}$ Carmine Della Vella, ${ }^{a}$ Rocco Schiavello, MD, ${ }^{b}$ and Gianfederico Possati, MDa

From the Departments of Cardiac Surgery ${ }^{\mathrm{a}}$ and Cardiac Anesthesia, ${ }^{\text {b }}$ Catholic University, Rome, Italy.

The authors had full access to the data and take responsibility for its integrity. All authors have read and agree to the manuscript as written.

Presented at the Fifth European Association for Cardio-thoracic Surgery/European Society of Thoracic Surgeons Joint Meeting, Stockholm, Sweden, September 9-13, 2006.

Received for publication Jan 18, 2007; revisions received April 16, 2007; accepted for publication April 20, 2007.

Address for reprints: Mario Gaudino, MD, Divisione di Cardiochirurgia, Policlinico Universitario A. Gemelli, Largo A. Gemelli 8, 00168 Rome, Italy (E-mail: mgaudino@ tiscali.it).

J Thorac Cardiovasc Surg 2007;134:465-9

$0022-5223 / \$ 32.00$

Copyright @ 2007 by The American Association for Thoracic Surgery

doi:10.1016/j.jtcvs.2007.04.028
Objective: Our objective was to evaluate the long-term survival and quality of life of patients who faced a prolonged ( $>10$ days) postoperative stay in the intensive care unit and were discharged from the hospital.

Methods: Among 3125 consecutive patients who underwent cardiac operations in a 5 -year period, we prospectively identified 57 who faced a prolonged postoperative intensive care unit stay and were discharged alive from the hospital. Patients were enrolled in a prospective follow-up protocol and evaluated every 6 to 12 months both clinically and instrumentally.

Results: Mean intensive care unit stay was $34 \pm 9$ days (range 11-141 days). Follow-up was complete and mean follow-up time was 71 months. Overall survival was $12(21 \%)$ of 57, and the majority of follow-up deaths were cardiac related. Of the surviving patients, only a small minority (4/12) regained full autonomy and returned to their previous lifestyle. Risk factors for prolonged intensive care unit stay were age, New York Heart Association/Canadian Cardiovascular Society class, hypertension, diabetes, low ejection fraction, aortic surgery, preoperative renal failure, nonelective surgery, prolonged cardiopulmonary bypass time, and perioperative use of aortic counterpulsator.

Conclusions: Patients who face a prolonged postoperative intensive care unit stay and who were discharged from the hospital have a very poor long-term outcome and even worse quality of life. These data lead to a consideration of the wisdom of using heroic treatment in patients who face a prolonged postoperative intensive care unit stay in view of the dismal clinical results and enormous use of hospital and human resources.

A prolonged postoperative stay of patients in the intensive care unit (ICU) is becoming more and more frequent in modern cardiac surgery, and this raises enormous clinical and ethical issues. However, to date, only scant information exists in the current literature on the outcome of patients with prolonged ICU stays, and most of the published series do not reflect the contemporary clinical reality. ${ }^{1,2}$

To provide some objective basis for clinical decision-making, we reviewed our recent experience with patients who faced a prolonged postoperative ICU stay (which we defined as $\geq 10$ days) and were discharged from the hospital, with particular attention to long-term outcome and identification of risk factors.

\section{Patients and Methods \\ Patient Population}

From January 1996 to December 2001, 3125 consecutive patients underwent major isolated cardiac surgery at our institution. Among them we identified 57 (1.8\%) who faced a 


\section{Abbreviation and Acronym}

ICU $=$ intensive care unit

prolonged ( $>10$ days) postoperative ICU stay and were discharged alive from the hospital; these patients represent the patient population for the present investigation.

Preoperative, intraoperative, and postoperative data of all patients were prospectively collected according to the definitions in use at our institution and reported in the appendix; all data were then entered in a computerized database.

Univariate and multivariate analysis to identify predictors of prolonged ICU stay were conducted on the overall population.

Follow-up. Each patient was followed up regularly at our institution 6 months after the operation and every year thereafter. At each time interval, clinical examination was performed and the results of surface electrocardiography, stress thallium 201 myocardial scintigraphy, 24-hour Holter monitoring, and transthoracic echocardiography were carefully reviewed. Invasive studies were proposed to the patients only in case of abnormal results of these first-line examinations.

The Karnofsky performance status (Table 1) was used to quantify the degree of dependence during everyday activity on follow-up.

For the purpose of the present study, all patients were resubmitted to clinical examination and all examinations were reviewed at the time of follow-up. In case of death, all available clinical data were collected and reviewed to establish the cause of the fatality. Death was considered cardiac in origin when it was preceded by objective evidence of cardiac dysfunction and noncardiac when a clear systemic or accidental cause of death was evident.

Follow-up was $100 \%$ complete and mean follow-up time was $71 \pm 13$ months.

\section{Statistical Analysis}

All data were included in an electronic database and processed with SPSS 10.1 for Windows software (SPSS, Inc, Chicago, Ill). Values of variables are expressed as mean \pm standard deviation for continuous variables or as percentage for discrete variables.

TABLE 1. Overview of the Karnofsky performance status

\begin{tabular}{lc}
\hline Performance & Karnofsky score \\
\hline No evidence of disease & 100 \\
Normal activity, minor signs of disease & 90 \\
Normal activity, signs of disease with effort & 80 \\
Self-care, unable to carry out normal activity & 70 \\
Assistance needed, able to care for most of & 60 \\
$\quad$ own needs & 50 \\
Considerable assistance required & 40 \\
Disabled, special care and assistance & \\
$\quad$ required & 30 \\
Severely disabled & 20 \\
Supportive treatment needed & 10 \\
Moribund & \\
\hline
\end{tabular}

TABLE 2. Preoperative characteristics of the patients who faced prolonged postoperative ICU stay

$\begin{array}{lc}\text { Mean age (y) } & 72 \pm 9 \\ \text { Male/female } & 38 / 19 \\ \text { Chronic pulmonary disease } & 9(15.8 \%) \\ \text { Extracardiac vasculopathy } & 21(36.8 \%) \\ \text { Diabetes } & 19(33.4 \%) \\ \text { Hypertension } & 17(29.8 \%) \\ \text { Previous cardiac surgery } & 16(28.1 \%) \\ \text { Serum creatinine }>2.0 \mathrm{mg} / \mathrm{dL} & 23(40.3 \%) \\ \text { NYHA/CCS class III-IV } & 19(33.4 \%) \\ \text { LVEF }<0.30 & 25(43.8 \%) \\ \text { Urgent/emergency operation } & 10(17.5 \%) \\ \text { Aortic surgery } & 19(33.4 \%) \\ \text { Mean CPB time (min) } & 154 \pm 31\end{array}$

ICU, Intensive care unit; NYHA/CCS, New York Heart Association/Canadian Cardiovascular Society; $L V E F$, left ventricular ejection fraction; $C P B$, cardiopulmonary bypass.

Baseline patient variables tested, applying multiple logistic regression analysis for association with the development of prolonged ICU stay in the overall population (3125 individuals), were as follows: age, sex, body surface area, New York Heart Association/Canadian Cardiovascular Society functional class, hypertension, diabetes, preoperative serum creatinine (milligrams per deciliter), platelets (number per nanoliter), left ventricular ejection fraction, pulmonary disease, extracardiac vasculopathy, intraoperative evidence of ascending aortic atherosclerosis, number of diseased coronary vessels, type of operation performed (coronary, valve, aortic, combined procedure), timing of operation (elective vs urgent/emergency), perioperative use of aortic counterpulsation, reoperation, time of operation, cardiopulmonary bypass time, crossclamp time, and year of operation. The assumptions of multiple regression were checked and met, and the model was validated through a "bootstrap" method. All potential explanatory variables were assessed for colinearity, and the explanatory variables were tested for interaction.

\section{Results}

During the study period, a total of 121 patients had a postoperative ICU stay longer than 10 days; 64 of them died in the hospital, and 57 survived and were discharged. These 57 patients constitute the basis of the present report; their main clinical and instrumental features are summarized in Table 2.

Mean ICU stay was $34 \pm 9$ days (range, 11-141 days).

Multiple logistic regression identified the following factors as predictors of prolonged ICU stay: age, advanced New York Heart Association/Canadian Cardiovascular Society class, hypertension, diabetes, elevated preoperative serum creatinine level, low left ventricular ejection fraction, urgent/emergency operation, perioperative use of aortic counterpulsator, prolonged cardiopulmonary bypass time, and aortic surgery (Table 3 ).

During the follow-up period, 45 (78.9\%) patients died (Figure 1). Causes of death are detailed in Table 4; 29 of 
TABLE 3. Predictors of prolonged ICU stay ( $>10$ days)

\begin{tabular}{llll}
\hline Variable & $\boldsymbol{\beta}$ & $\mathbf{O R}$ & $\boldsymbol{P}$ value \\
\hline Age $>70$ y & 1.435 & 2.9 & $\mathbf{. 0 3 7}$ \\
Advanced NYHA/CCS class* & 2.08 & 5.91 & $\mathbf{. 0 1 5}$ \\
Systemic hypertension & 2.26 & 1.98 & $\mathbf{. 0 2 2}$ \\
Diabetes & 1.897 & 3.71 & $\mathbf{. 0 0 9}$ \\
Preoperative creatinine level $>2 \mathrm{mg} / \mathrm{dL}$ & 2.51 & 2.58 & $\mathbf{. 0 3 7}$ \\
LVEF $<50 \%$ & 3.055 & 13.2 & $\mathbf{. 0 0 3}$ \\
Nonelective surgery & 2.807 & 9.61 & $\mathbf{. 0 0 2}$ \\
CPB time $>180$ min & 1.206 & 1.9 & $\mathbf{. 0 3 5}$ \\
Aortic surgery & 3.501 & 9.87 & $\mathbf{. 0 2 8}$ \\
Perioperative aortic counterpulsation & 1.73 & 5.0 & $\mathbf{. 0 0 1}$
\end{tabular}

Multiple logistic regression model in the overall unselected population. Boldface values indicate statistical significance. ICU, Intensive care unit; $O R$, odds ratio; $L V E F$, left ventricular ejection fraction; NYHA/CCS, New York Heart Association/Canadian Cardiovascular Society; CPB, cardiopulmonary bypass. *NYHA III or IV and/or CCS III or IV.

these deaths were judged to be cardiac in origin. Among the surviving patients, only a small minority (4/12) regained full autonomy and returned to their previous lifestyle (Karnofsky score $>80$ ); 2 patients were never discharged from the rehabilitation facility and the remaining 6 were highly dependent on external support for all everyday activities (Karnofsky score $<50$ ). Table 5 stratifies the surviving patients on the basis of the quality of life during the 2002-2006 follow-up period. At all time points, most patients had a low Karnofsky score $(<50)$, and only a minority of individuals had an intermediate $(<80$ and $>50)$ or high $(>80)$ score. Additionally, there were no improvements in the score of individual survivors over time.

\section{Discussion}

In the current era, the progressive worsening of the risk profile of patients referred for cardiac operations, coupled with the amelioration of the surgical results and of the efficacy of the intensive medical treatment, has rendered prolonged postoperative ICU stays more and more common.

These patients elicit major clinical as well as ethical issues. They usually require extraordinary human and economic resources, thus accounting for a considerable part of
TABLE 4. Causes of death during follow-up

\begin{tabular}{lc}
\hline Cause of death & No. \\
\hline Myocardial infarction & 7 \\
Pulmonary embolism & 2 \\
Aortic aneurysm & 5 \\
Aortic dissection & 2 \\
Arrhythmias & 4 \\
Heart failure & 9 \\
Cancer & 5 \\
Others & 11 \\
\hline
\end{tabular}

the overall departmental costs in terms of both clinical assistance and economic use. In a recent series, Williams and colleagues ${ }^{1}$ showed that their 49 patients with ICU stays longer than 14 days consumed one third of all ICU bed days for the entire year.

Generally speaking, survival is better for cardiac versus general surgical patients with prolonged ICU stays. ${ }^{3-5} \mathrm{Nev}$ ertheless, a significant proportion of these patients finally die after a long and costly hospitalization. ${ }^{1,3-6}$ In addition, little is known about the long-term fate of these patients after successful hospital discharge, since most of the reported series are outdated and do not reflect the contemporary practice. ${ }^{1,3-8}$

Although physicians should not deny potentially successful treatments to these desperately ill patients and should preserve their lives until the very end, we should also avoid inappropriate use of clinical and economic resources that could be allocated for other patients still on the waiting list for surgery.

With the aim of contributing to the knowledge on this complex issue and to provide an objective basis for clinical decision-making, we reviewed our recent experience with patients who faced a prolonged ( $>10$ days) postoperative ICU stay after cardiac surgery and were discharged alive from the hospital. To most surgeons and anesthesiologists, these very few cases are supposedly a clinical success.

Overall, we had dismal clinical results. At a mean follow-up that approximates 6 years, almost $80 \%$ of patients have died

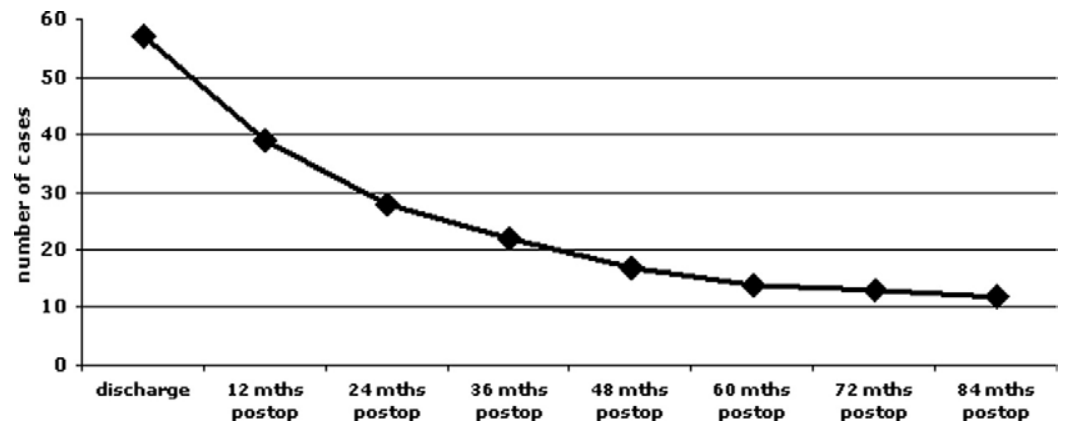

Figure 1. Postoperative survival. 
TABLE 5. Quality-of-life results of survivors in the follow-up period 2002-2006 (Karnofsky performance status score)

\begin{tabular}{|c|c|c|c|c|c|}
\hline & Year 2002 & Year 2003 & Year 2004 & Year 2005 & Year 2006 \\
\hline Patients with score $>80$ (N) & 8 & 6 & 5 & 4 & 4 \\
\hline Patients with score $<80$ and $>50$ (N) & 9 & 7 & 5 & 3 & 2 \\
\hline Patients with score $<50$ (N) & 21 & 18 & 12 & 9 & 6 \\
\hline
\end{tabular}

(the majority from cardiac causes) and the few survivors have very poor quality of life. Only $4(7 \%)$ of 57 patients had a Karnofsky score greater than 80 (that is, they regained full autonomy and returned to their previous lifestyle).

In a previous investigation Hellgren and Stahle ${ }^{6}$ observed a $68 \%$ survival at a 5 -year follow-up in a population of patients whose ICU stay lasted more than 8 days. After a 2-year follow-up, Williams and coauthors ${ }^{1}$ reported 22 patients surviving of 49 who faced an ICU stay longer than 14 days; 16 of these had "a normal quality of life." It is difficult to reliably compare the experiences in this field owing to different criteria of inclusion of patients and different methods of evaluation of quality of life. Anyway, all studies indicate that both survival and quality of life are significantly worse than those seen among individuals who had shorter ICU stays.

As physicians, we are prompted to consider as a clinical success the discharge of a living patient after a prolonged and complicated ICU stay. The current findings suggest that in many cases this idea is misleading, inasmuch as the outcome of these patients is very poor. Raw mortality and morbidity rates inadequately describe the fate of this subgroup of patients, although the end of follow-up mortality, which approaches $80 \%$, is per se highly indicative.

Quality-of-life measurements more accurately reflect the extent of our success. It is sad to note that, after major efforts have been undertaken to discharge these patients alive, the Karnofksy score remains radically impaired even in the scarce survivors.

The consideration that the quality of life of these patients is very poor after hospital discharge and does not improve during the follow-up period further suggests that in this setting heroic measures represent poor resource allocation.

The data presented herein unmask an apparent success as a true clinical failure. The patients who are discharged alive from the hospital represent only a small minority of the overall population of individuals who have prolonged ICU stay. In fact, most of these will die in the ICU or even in the hospital. ${ }^{8}$ Those who finally leave the hospital alive constitute a fraction of this population and a very select subgroup, but their discharge cannot be considered as an achievement. The majority of them will die out of the hospital, and the survivors will remain profoundly limited in their lifestyle and often highly dependent on external support for any daily activity.
In our opinion, these data should lead to a reconsideration of the wisdom of attempting heroic treatment in cardiac surgical patients who face a prolonged postoperative ICU stay.

It is not our intent to furnish a solution for such a complex problem, but to emphasize its proportions and its potential to grow dramatically in the coming years. More studies are needed, and it seems unlikely that such complex cases will never have simple answers. Nonetheless, the very poor clinical results and the enormous use of hospital and human resources (with consequent delay and complications for those on the waiting list for surgery) cannot be ignored.

\section{References}

1. Williams MR, Wellner RB, Hartnett EA, Thornton B, Kavarana MN, Mahapatra R, et al. Long-term survival and quality of life in cardiac surgical patients with prolonged intensive care unit length of stay. Ann Thorac Surg. 2002;73:1472-8.

2. Bapat V, Allen D, Young C, Roxburgh J, Ibrahim M. Survival and quality of life of cardiac surgery complicated by prolonged Intensive Care. J Card Surg. 2005;20:212-7.

3. Yusuf S, Zucker D, Peduzzi P, Fisher LD, Takaro T, Kennedy JW, et al. Effect of coronary artery bypass graft surgery on survival: overview of 10-year results from randomised trials by the Coronary Artery Bypass Graft Surgery Trialists Collaboration. Lancet. 1994;344:563-70.

4. Roques F, Nashef SA, Michel P, Gauducheau E, De Vincentiis C, Baudet E, et al. Risk factors and outcome in European cardiac surgery: analysis of the Euro-SCORE multinational database of 19,030 patients. Eur J Cardiothorac Surg. 1999;15:816-22.

5. Combes A, Costa MA, Trouillet JL, Baudot J, Mokhtari M, Gibert C, et al. Morbidity, mortality, and quality-of-life outcomes of patients requiring $>$ or $=14$ days of mechanical ventilation. Crit Care Med. 2003;31: 1373-81

6. Hellgren L, Stahle E. Quality of life after heart valve surgery with prolonged intensive care. Ann Thorac Surg. 2005;80:1693-8.

7. Wong DT, Cheng D, Kustra R, Tibshirani R, Karski J, Carroll-Munro J, et al. Risk factors of delayed extubation, prolonged length of stay in the Intensive Care Unit, and mortality in patients undergoing coronary artery bypass graft with fast-track cardiac anesthesia, Anesthesiology. 1999;91:936-44.

8. Hein OV, Birnbaum J, Wernecke K, England M, Konertz W, Spies C. Prolonged Intensiva Care Unit stay in cardiac surgery: risk factors and long-term survival. Ann Thorac Surg. 2006;81:880-5.

\section{Appendix \\ Definitions}

Extracardiac vasculopathy: Monolateral or bilateral carotid stenosis of $70 \%$ or more, clinical or instrumental evidence of lower limb or aortic atherosclerosis, or previous cerebral vascular episode.

Myocardial infarction: Diagnosed on the basis of echocardiographic evidence of regional hypokinesia or dyskinesia, MB fraction greater than $4 \%$ of the total hematic level of creatine kinase 
concentration, and appearance of new Q waves on the electrocardiogram.

Renal insufficiency: Serum creatinine greater than $2.0 \mathrm{mg} / \mathrm{dL}$.

Respiratory insufficiency: Arterial oxygen tension less than $60 \mathrm{~mm} \mathrm{Hg}$ in room air.

Chronic obstructive respiratory disease: Long-term $(\geq 6$ months) use of bronchodilators or steroids.

Intraoperative stroke: A new focal neurologic deficit or coma associated with computed tomographic demonstration of recent ischemic cerebral lesion, which became evident at the moment the patient awakened from the anesthesia and lasted more than 24 hours.
Postoperative stroke: A new focal neurologic deficit or coma associated with computed tomographic demonstration of recent ischemic cerebral lesion and lasting more than 24 hours, which became evident after a normal awakening of the patient from the anesthesia and a normal postoperative neurologic status.

Major postoperative complications: Death, stroke, shock, sepsis, myocardial infarction, reoperation.

Minor postoperative complications: Renal insufficiency, mechanical ventilation for more than 24 hours, respiratory insufficiency, inotropic support for more than 24 hours, need for blood transfusions, revision for bleeding. 